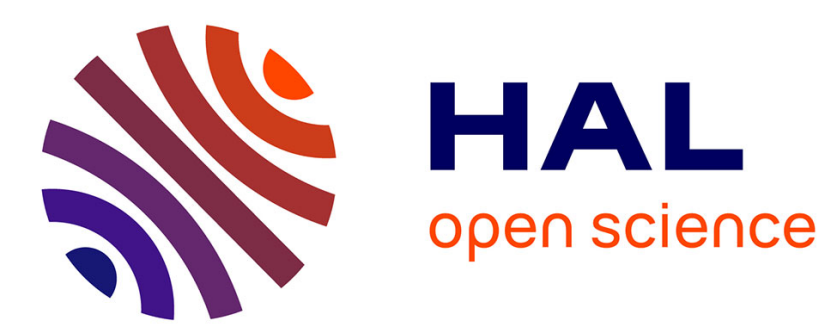

\title{
Coordination pédagogique : une vision globale de l'accompagnement personnalisé au DESS DICIT
}

Lydie Réné-Boullier

\section{To cite this version:}

Lydie Réné-Boullier. Coordination pédagogique : une vision globale de l'accompagnement personnalisé au DESS DICIT. STICEF (Sciences et Technologies de l'Information et de la Communication pour l'Éducation et la Formation), 2003, 10, 15 p. hal-00696402

\section{HAL Id: hal-00696402 \\ https://hal.science/hal-00696402}

Submitted on 11 May 2012

HAL is a multi-disciplinary open access archive for the deposit and dissemination of scientific research documents, whether they are published or not. The documents may come from teaching and research institutions in France or abroad, or from public or private research centers.
L'archive ouverte pluridisciplinaire HAL, est destinée au dépôt et à la diffusion de documents scientifiques de niveau recherche, publiés ou non, émanant des établissements d'enseignement et de recherche français ou étrangers, des laboratoires publics ou privés. 


\section{Coordination pédagogique : une vision globale de I'accompagnement personnalisé au DESS DICIT}

\section{Lydie Réné-Boullier [Université de Technologie de Compiègne]}

- RÉSUMÉ : Le coordinateur pédagogique au DESS DICIT a une vision globale de la promotion, qu'il suit sur toute la durée de la formation. Garant des règles et des principes pédagogiques de l'institution, il coordonne les enseignants, conseille et encourage les étudiants. L'accompagnement personnalisé des étudiants, soutenu par la planification des scénarios pédagogiques, permet de combattre les abandons et d'aider les étudiants à se structurer.

- MOTS-CLÉS : coordination pédagogique, accompagnement, encadrement, structuration, abandon.

\section{Introduction}

Dans le contexte actuel, le développement des technologies de l'information et de la communication pour l'éducation (TICE) est considéré comme une obligation pour élargir l'accès à la formation. Les technologies nouvelles transforment à la fois les dispositifs, le rapport au savoir, les dynamiques d'apprentissage et permettent une industrialisation de certains éléments. Mais leur introduction n'a de sens que si elle permet de rationaliser les coûts - pour assurer la pérennité et les résultats de la formation tout en affirmant, la responsabilité pédagogique de l'institution enseignante et en garantissant la qualité pour l'usager.

\subsection{L'étudiant "virtuel"}

Les critères de qualité sont à déterminer en fonction de cet "usager", mais les étudiants ont du mal à percevoir ce monde nouveau de la formation à distance et les bouleversements qui vont en découler. Ils ont d'autant plus de mal à définir a priori leurs attentes. C'est donc aux porteurs du projet de définir les règles pédagogiques et organisationnelles et la juste prise en charge des étudiants tout au long de la formation. Comme le souligne Guy Le Bouëdec [LeBouëdec02 $]^{[1]}$, cette responsabilité de l'institution et du pédagogue ne doit pas être obscurcie par le principe d'autonomie de l'étudiant, qui reste un mot vide de réalité.

En effet, quelle forme prend l'autonomie dans la réalité ? Chaque étudiant se sent plus ou moins à l'aise sur une ou plusieurs techniques ou matières mais l'autonomie universelle qui s'apparente fort à l'autarcie est contraire à tout projet pédagogique, construit pour et vers l'étudiant. L'accompagnement nécessaire à la progression pédagogique peut être plus ou moins prégnant suivant le degré d'expertise des étudiants, mais il se doit toujours d'exister. Le Massachusset Institut of Technology (MIT) ne s'y pas trompé quand il laisse à disposition ses contenus gratuitement sur le web. Il fait payer les services mis en œuvre pour accompagner les étudiants, qui permettent l'élaboration de la construction du savoir, et non les contenus. La plus grande Bibliothèque ne façonne que les autodidactes avérés!

Cette exigence de l'autonomie, laissant l'homme seul face à la machine, est peut-être une des raisons des taux d'abandon de 50 et $70 \%$ dans certaines FOAD, alors que dans d'autres, ils tournent entre 5 et $15 \%$. 
Ces forts taux d'abandon laissent à penser que les étudiants ont des raisons communes de quitter les formations. Les entretiens que nous avons conduits, dans le cadre d'une recherche en cours ${ }^{[2]}$, ont permis de mettre à jour des réalités qui peuvent paraître évidentes mais qui sont souvent occultées :

- L'étudiant est une personne qu'on ne peut réduire à son rôle unique d'étudiant car la formation s'inscrit dans son évolution (dû au processus de formation), sa vie quotidienne et sociale. Il faut le regarder comme une personne entière et ne pas considérer que son temps d'apprentissage peut exister en dehors sa vie sociale. L'un et l'autre vont s'influencer.

- Les espaces/temps de formation se font aux croisements de différents univers sociaux (Travail à domicile/vie personnelle - Formation sur le poste de travail) qui induisent des sauts d'un rôle social à l'autre et créent des tensions.

- L'étudiant doit mobiliser des ressources et des compétences multiples que la situation à distance oblige à trouver en soi, alors que la formation classique permettait une répartition entre - et avec - les enseignants et les pairs. Ainsi l'étudiant doit à la fois gérer son temps et ses activités, diagnostiquer ses faiblesses, demander de l'aide, se débrouiller avec la technologie et accepter avec tolérance les pannes techniques...

- L'isolement dans la formation ouverte et à distance (FOAD) est vécu difficilement. Chaque doute sur l'organisation, sur un énoncé, sur une date devient problème si la réponse n'est pas rapide, car l'enseignant n'est pas là au moment où la question se pose. L'apport pédagogique du groupe n'est plus, ou n'est plus immédiat, ce qui freine la progression de la pensée, la contradiction toujours favorable à la réflexion et oblige à une constante motivation personnelle.

Le travail de l'étudiant à distance est donc plus complexe que celui de l'étudiant en mode présentiel car il ne peut pas s'appuyer sur un groupe (enseignants et pairs) et ni évoluer à l'intérieur des repères sociaux d'une formation classique (classes-horaires...) pris en charge par l'institution.

\subsection{L'enseignant multi-tâches}

Face à ces obligations nouvelles de l'étudiant à distance se dessinent celles de l'enseignant. Il doit à la fois être expert du contenu, de la technologie (logiciels adéquats/plateforme), et de l'édition des supports. Mais il doit aussi devenir un scénariste ayant la maîtrise des mécanismes de l'encadrement à distance, et un suiveur sachant à la fois communiquer, encadrer, gérer les groupes. Il n'est plus seulement l'expert du contenu, il doit aussi connaître la réalité de la formation des adultes. Même avec une équipe pédagogique qui les soutient, les enseignants ressentent ce passage vers la formation distance, comme une remise en question de leur profession et de leur rôle.

Des choix institutionnels surgissent : doit-on donner la charge des cours en face à face aux enseignants et le tutorat à des tuteurs comme c'est le cas entre cours et travaux dirigés ? Doit-on séparer l'enseignement et le contenu ? Ou encore doit-on, pour faire baisser les coûts, confier le contenu à un enseignant-auteur et la forme à un éditeur garant des règles de lisibilité et de structuration (ou spécialiste des outils interactifs) ?

Durant chaque projet, toute l'équipe pédagogique doit mobiliser ses ressources et son imagination afin que chacun trouve un rôle à sa mesure. Rapidement, en général, un nouveau membre apparaît : le tuteur. Il prend en charge la correction des travaux à distance et l'animation des forums en partenariat avec l'enseignant. Puis petit à petit, pour réguler l'ensemble d'une formation dans ses échanges, le rôle de coordination pédagogique s'impose comme une interface humaine, garante des règles de l'institution mais à l'écoute des enseignants, des tuteurs et des étudiants.

À travers le cas particulier du DESS DICIT, nous allons vous présenter les rôles que nous avons définis pour ces deux derniers protagonistes de l'histoire de la formation à distance. 


\section{Le cadre particulier du DESS DICIT}

À l'Université de technologie de Compiègne, le DESS DICIT (Diplôme en Ingénierie de Communication Technologique et Industrielle), a été le premier cursus complet à distance de l'Université dès 1995. Il comportait 15 unités de valeur avec 13 enseignants/tuteurs. Il accueillait des personnes en formation continue et des demandeurs d'emploi ${ }^{[3]}$. La rédaction technique n'étant pas un aboutissement de filière, les étudiants avaient tous des formations et des expériences différentes: scientifiques, techniques ou littéraires.

Le projet des responsables n'était pas de créer une formation "ouverte" et à distance où chacun pourrait se connecter à volonté, sans contrainte ni guidance. Au contraire, la formation ne devait mériter l'adjectif d'ouverte qu'à la condition d'être "ouverte" à tous. Elle se devait d'accueillir à la fois les étudiants déterminés et ceux, hésitants face à la formation à distance ou à la technique, qui possèdent pourtant les qualités professionnelles et humaines nécessaires au métier. Les interactions avec l'équipe pédagogique allaient donc être fréquentes pour permettre à tous d'avancer malgré leurs différences, au même rythme et avec les mêmes chances.

Par ailleurs, la rédaction technique demande à la fois de l'autonomie et une aisance dans le travail en groupe. Le projet pédagogique des responsables visait donc l'apprentissage d'un métier complexe nécessitant à la fois le maniement de concepts, de méthodes, de techniques et une grande facilité à communiquer. Pour y arriver, des choix pédagogiques avaient été cadrés et certains nécessitaient d'établir des règles strictes de suivi :

- Un parcours complet, appuyé sur une progression pédagogique conçue à la fois par les responsables et les professionnels du métier associés au projet. Ce parcours obligeait le groupe à avancer au même rythme, pour suivre les modules dans le même ordre au même moment...

- Une scénarisation précise des travaux avec une date de remise butoir. Cette rigueur nécessitait un suivi pour les étudiants et les enseignants. Les corrections étaient attendues avec impatience, pour éclairer la mise en œuvre de l'exercice suivant.

- Des exercices à réaliser en groupe. Ces travaux requéraient un médiateur ponctuel et réactif (qui pouvait intervenir aussi en cas de problème entre un étudiant et un enseignant/tuteur) tandis que l'évaluation du groupe et de son avancement demandait un suivi quotidien.

- La technique devait permettre au projet pédagogique institutionnel de se mettre en forme (et non le contraire). C'est une des raisons qui nous ont amenés à choisir la plateforme Learning-Space de Notes. En effet, cette plateforme très structurée permettait :

- de s'appuyer sur des modèles virtuels correspondant aux pratiques de formation présentielle - salle de classe, cahier de texte, agenda, médiathèque, carnet de note - pour retrouver des habitudes,

- de créer des repères visuels (voir figure 1) correspondant à nos temps de formation et de regroupements, mais aussi aux temps forts des différentes Unités de valeur,

- d'afficher l'organisation des Unités de valeur en détaillant leur scénarisation, de suivre les étudiants personnellement, de permettre des travaux de groupe (voir figure 2) avec un suivi chronologique des actions

(sous forme de forum),

- de renvoyer des corrections personnalisées et collectives.

Sur la plateforme, pour chaque matière, les activités des étudiants sont scénarisées dans la base Programme. L'interface de cette base est présentée en fonction de l'organisation du temps réel : 3 présentiels et 2 périodes à distance. 


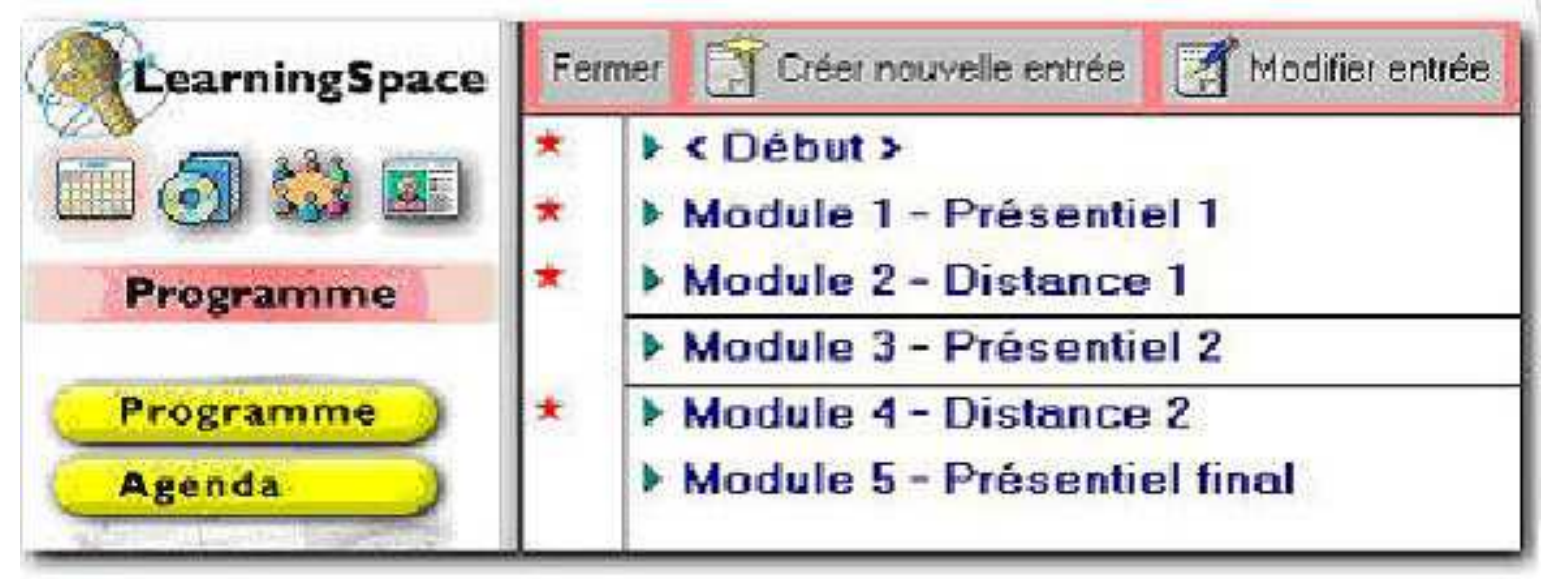

Figure 1 : Interface du programme par matière (agenda des travaux à rendre)

Le suivi du forum permet de voir si tout le groupe participe régulièrement. Les outils offerts nous convenaient puisqu'il était bien question de prendre en charge nos étudiants, de les suivre, de les contrôler et de les aider au moment où la motivation pouvait baisser. Il était donc essentiel de pouvoir suivre le travail de chacun ${ }^{[4]}$.

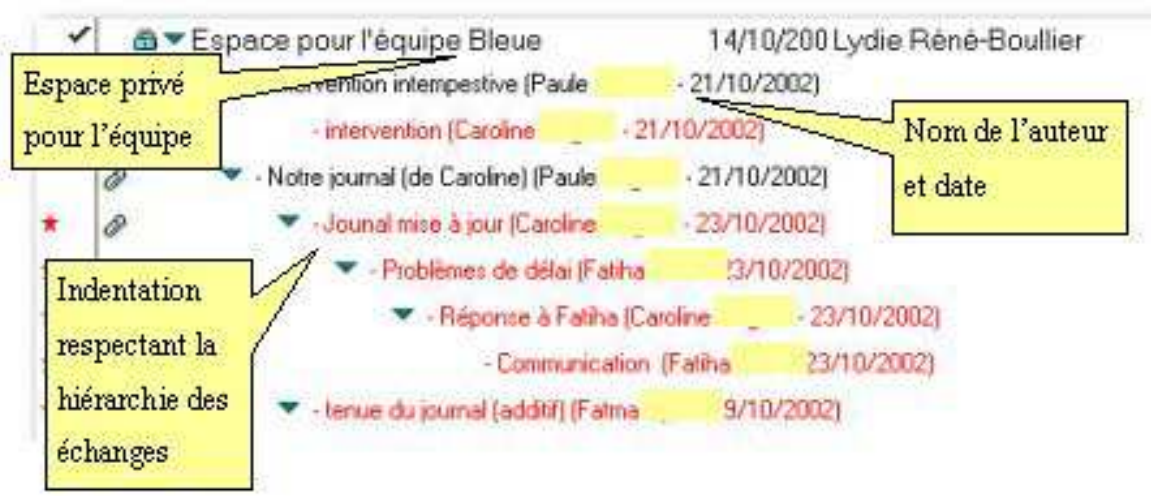

Figure 2 : Travail collaboratif par le forum asynchrone

Dès le départ, nous avons défini précisément les rôles et les obligations pour chacun des acteurs de la formation: responsable du projet, responsable pédagogique, administrateur, enseignants, tuteur. Puis après les premiers mois, en réponse à une demande des enseignants et des étudiants, nous avons élaboré un rôle particulier de coordination pédagogique pour prendre en charge la gestion des échanges de la formation dans sa globalité. Cette fonction, qui n'est reconnue dans aucune plate-forme, semble aujourd'hui de plus en plus reproduite dans les formations à distance. Sa définition s'est finalisée au fur et à mesure de la première année.

\subsection{Le rôle de tuteur}

Le DESS est un diplôme professionnel de rédaction technique, de ce fait la moitié des enseignants sont des gens de terrain qui ont un emploi dans la spécialité, en plus d'être enseignant. L'autre moitié est composée d'enseignants d'université qui ont d'autres fonctions. La plupart assurent formation en face à face et correction à distance. Pourtant, dès la troisième année, une fois les scénarisations misent au point et les exercices et travaux cadrés, certains ont souhaité avoir recours à un tuteur pour se dégager du temps. Cette fonction de tutorat est donc occupée soit par enseignants, soit par des tuteurs mais c'est bien le rôle de tuteur dans notre formation à distance qu'il s'agit ici de définir, qu'il soit ou non doublé du rôle d'enseignant. 
Nous avons choisi comme tuteurs (au sens strict) des anciens du DESS pour des raisons qui nous semblent essentielles. Ils sont venus se former à l'université en formation continue, ils ont déjà une connaissance parlante du métier. Ils ont été repérés pour la qualité de leurs travaux, choisis par un enseignant et acceptés par les responsables. Ils connaissent notre plate-forme, toute l'équipe pédagogique et ils ont assimilé nos règles et notre organisation.

Cela permet à nos tuteurs :

- de comprendre plus facilement la tâche à effectuer par les étudiants car ils ont été confrontés aux travaux qu'ils proposent,

- d'avoir une idée de la charge de travail effective du fait que plusieurs matières sont réalisées en parallèle (les enseignants ont moins cette vision globale de la charge de travail),

- d'interpréter les signes de lassitude ou de non-compréhension car ils ont vécu aussi cette surcharge cognitive due à la formation (travail en entreprise ou stage et formation) et l'ont partagée avec leurs pairs.

Avant la formation, le tuteur doit organiser le travail, évaluer le temps de réalisation effectif des travaux. Il doit aussi s'il le peut clarifier le contenu. En effet, chaque année, il faut revenir sur l'organisation d'un module, préciser un énoncé... Les tuteurs assistent en partie aux cours en face à face. Cela leur permet d'avoir connaissance de ce qui a été dit. De plus, la rencontre avec les étudiants est essentielle pour amorcer la communication à distance et se faire une première idée des différentes personnalités du groupe. Il sera ensuite plus facile d'entrer en communication avec l'étudiant.

Pour les corrections, un tuteur est toujours positif même s'il fait apparaitre les points à faire évoluer, et les erreurs. Il doit créer un environnement d'apprentissage amical, surtout pour les travaux de groupe où il est essentiel de maintenir l'unité. C'est là qu'il devient psychologue : il encourage telle ou telle personne à prendre plus de part dans un débat, à freiner son ardeur ou son vocabulaire, à ne pas garder le leadership.

Le tuteur a un vrai rôle pédagogique. Il doit être au fait du contenu pour faire des corrections pointues et régulières, car l'évaluation en cours d'apprentissage est importante pour que l'étudiant progresse. Les corrections sont obligatoirement personnalisées et détaillées pour permettre à l'étudiant de reprendre son travail en sachant comment l'améliorer. Un corrigé type peut être offert en supplément. C'est aussi au tuteur que revient la charge de traquer la triche, en comparant les fichiers, les auteurs, les styles... Pour l'examen final, en classe et sur machine, la présence de l'enseignant et les heures de "dépose » des fichiers sur le serveur restent les garants de la sécurité pour la qualification finale.

Il est à noter que nous avons enlevé au tuteur le rôle technique qui lui est parfois attribué, car notre administrateur réseau fait office de hot-line informatique. Cependant, le tuteur pourrait rapidement se transformer en hot-line pédagogique ouverte 7 jours sur 7, et 24 heures par jour, puisque le courriel fonctionne en permanence. Heureusement cette assiduité ne lui est pas demandée car, rappelons-le, le tuteur est aussi un professionnel qui a d'autres obligations. Les règles de réponse pour des questions de cours sont d'un à deux jours (hors week-end); par contre, il est impératif que les corrections arrivent dans les 3 jours aux étudiants (week-end compris). Cela ne pose en général pas de problème puisque les tuteurs proposent, eux-mêmes, leurs jours de corrections au coordinateur pédagogique qui gère le planning global de la formation.

La fonction du tuteur est souvent sous-évaluée alors qu'elle nécessite de mettre en pratique de multiples compétences. Au DICIT, leur fonction est reconnue, ils font partie de l'équipe pédagogique, et sont payés sur le même barème que les enseignants. Pourtant ils se sentent parfois isolés. Le coordinateur pédagogique intervient alors pour aménager leur travail, les motiver, les écouter et leur permettre de gérer chaque étudiant individuellement. 


\subsection{Le rôle de coordinateur pédagogique}

À la différence de certains intervenants, les autres fonctions du coordinateur sont secondaires, il est le soutien de la promotion en cours et reste donc joignable, 7 jours sur 7 , de $9 \mathrm{~h}$ à $22 \mathrm{~h}$, pour répondre aux problèmes de tous.

\subsubsection{Souvent en amont avec les enseignants}

En ce qui concerne les enseignants, ils ont des attentes précises. La formation à distance les oblige à se remettre en question et à faire évoluer leurs manières d'enseigner. Ils veulent discuter des changements d'ordre pédagogique à opérer. Ils ont besoin de soutien pour la composition et la présentation des cours sous forme électronique, d'une aide dans la rédaction de leurs scénarii pédagogiques et enfin pour la mise en place des documents sur la plate-forme. Ils s'attendent à être dépannés, à recevoir une réponse rapide à leurs appels au secours. De plus, ils préparent chacun leur module sans visibilité globale de la formation ou avec une disponibilité réduite pour rencontrer les autres enseignants. Ils éprouvent, eux aussi, le besoin d'une personne ressource qui leur consacre du temps, les assiste, les conseille et les coordonne.

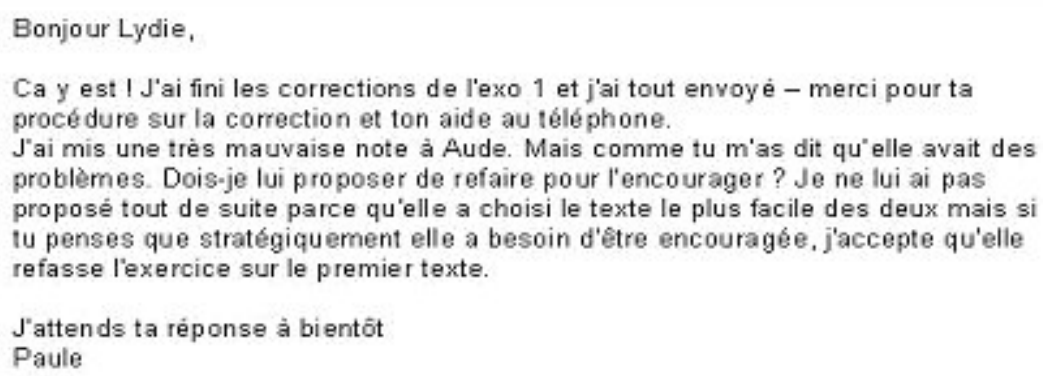

Figure 3 : Courriel d'une tutrice pour faire le point sur les premières corrections

Le plus délicat est d'établir des relations de confiance. Les enseignants ne doivent pas voir le coordinateur comme un "contrôleur" mais comme un conseiller même s'il a regard sur leur travail. Il est donc important de prendre le temps de discuter avec eux de la scénarisation de leurs cours, des relations avec les tuteurs, des moyens de correction et des règles de vie de la formation à distance. Le premier contact permet de mieux connaître leur projet, de pouvoir les assister au besoin. Le coordinateur aide les enseignants pour la prise en main des outils informatiques (plate-forme, logiciels) puis au dépannage rapide sur le site quand les liaisons techniques sont perturbées (pour mettre un fichier sur la base, pour ouvrir une entrée d'exercice à leur place...). Quand il connaît les contenus et que les enseignants ne sont pas joignables, il peut aussi répondre aux étudiants en cas d'urgence.

\subsubsection{Dès le début avec les tuteurs}

Les enseignants-tuteurs, avant le début de la formation, proposent leur jour de correction au coordinateur qui prépare le planning global. Le coordinateur estime avec chacun la charge de travail demandée aux étudiants afin d'espacer les travaux, dans une même matière, mais aussi ne pas entraîner une surcharge sur plusieurs matières, ceci en prenant en compte des desiderata d'organisation personnelle des tuteurs.

Afin de respecter les règles de correction, les tuteurs se tournent vers le coordinateur quand ils pressentent qu'ils ne pourront pas tenir une date de correction, ils obtiennent alors un délai que le coordinateur transmet aux étudiants. Comme les étudiants sont pénalisés sur leurs notes s'ils prennent du retard, sans donner une excuse valable au préalable, la règle s'applique aux tuteurs qui sont pénalisés financièrement s'ils n'avertissent pas dans les temps. Ce passage obligé par le référent institutionnel laisse une trace qui permet de régler les futurs problèmes de notation ou de paiement. 
De même, le coordinateur, qui a en charge le moral et la motivation des étudiants, qui reçoit les doléances et les excuses, se doit d'avertir le tuteur le plus rapidement possible quand un problème se fait jour. Ainsi celui-ci peut rectifier son action (rendre un corrigé type plus tard, par exemple !) pour ne pas pénaliser un étudiant qui a une excuse valable. Cet échange peut intervenir plus simplement quand le coordinateur sent une baisse de motivation ou une attente de suivi plus forte de la part d'un étudiant, ce qui permet au tuteur d'être plus attentif. De la même façon, dans son rôle de casque-bleu entre les enseignants-tuteurs et les étudiants, le coordinateur peut avoir à intervenir pour calmer une relation qui prend feu entre les deux parties. Cette intervention est souvent délicate car les échanges à distance sont perçus comme froids et sans autre vecteur de communication situé, plus à même de nuancer les propos. Comme ils portent sur un sujet brûlant "le travail et la mise en œuvre des capacités de l'étudiant" ces échanges peuvent rapidement s'enflammer sur la base d'un malentendu. Il faut alors beaucoup de diplomatie pour ramener le calme.

Ainsi, enseignants et tuteurs s'appuient sur le travail du coordinateur, lui font confiance et n'hésitent pas à échanger fréquemment avec lui malgré son rôle de pseudo-surveillant.

\subsubsection{Toujours avec les étudiants}

La rédaction technique ne demande pas un parcours antérieur imposé et de nombreux professionnels peuvent y trouver une réinsertion à partir d'une spécialisation première, restée sans débouché. Notre public est donc hétérogène par la filière, l'âge, l'expérience, les connaissances, les compétences et la socialisation. Il est essentiel pourtant de créer une vraie promotion dont les membres soient solidaires. C'est le travail du coordinateur.

Ces différences, qui font la richesse des promotions, ne sont pas toujours simples à gérer. Les étudiants, qui sortent de maîtrise, ont une habitude des exercices de type universitaire mais de réelles difficultés à appréhender le monde de l'entreprise. Les professionnels ont plus de mal avec les concepts et parfois même avec la technique. Quant aux demandeurs d'emploi (surtout ceux de longue durée), c'est la formation en elle-même qui est un souci quotidien même quand elle les aide, au bout du compte, à se réinsérer. Ils doivent retrouver à la fois une identité, une volonté, une organisation pour le travail en solo et faire face à tous les problèmes de vie ordinaire. Ces publics ont donc des attentes différentes mais tous sont demandeurs d'une écoute empathique. Le coordinateur est là pour leur répondre, sur le stage, sur l'organisation de la formation ou sur les évaluations, sans pour autant déranger les enseignants-tuteurs, qui ne sont pas obligatoirement disponibles toute la journée.

Les périodes de formations en face à face sont un atout, outre le fait qu'elles permettent de créer la synergie de groupe, elles restent un moment important de la formation à distance. En plus du recadrage avec les enseignants, ces périodes permettent au coordinateur de voir évoluer le groupe et ses réseaux, de prendre part et d'organiser sa vie (fêtes, repas) et ses rituels. Les anniversaires, comme le souligne Christian Helson [Helson03] sont des moments importants tant sur le plan collectif qu'individuel. Le tableau 1 permet de préparer, avec les intéressés, les fêtes qui souderont la promotion durant les regroupements.

Les enseignants-tuteurs suivent les progrès des étudiants pour leur matière mais n'ont pas une vision globale des personnes. C'est le coordinateur qui prend en charge le groupe, dans sa diversité, en tenant compte de ses particularités, sur toute la longueur de la formation. C'est lui qui écoute les problèmes de chacun, qui les soutient aussi bien le week-end que le soir quand ils se mettent enfin sur leur ordinateur, après le travail. 


\begin{tabular}{|l|l|l|l|l|}
\hline Prénom & Dates de naissance & Ages & & \\
\hline Audrey & $24 / 06 / 80$ & 22 ans & Juin & 6 \\
\hline Christine & $18 / 07 / 64$ & 38 ans & Juillet & 7 \\
\hline Sophie & $27 / 10 / 68$ & 34 ans & Oct & 10 \\
\hline Patrick & $15 / 12 / 55$ & 47 ans & Déc & 12 \\
\hline Aude & $23 / 06 / 79$ & 23 ans & Juin & 6 \\
\hline Anaïs & $08 / 05 / 64$ & 38 ans & Mai & 5 \\
\hline Caroline & $25 / 02 / 55$ & 47 ans & Fév & 2 \\
\hline Jean-Pierre & $07 / 09 / 59$ & 44 ans & Sept & 9 \\
\hline Caroline & $20 / 07 / 72$ & 30 ans & Juillet & 7 \\
\hline Pascal & $24 / 07 / 77$ & 25 ans & Juillet & 7 \\
\hline Charles & $17 / 05 / 72$ & 30 ans & Mai & 5 \\
\hline
\end{tabular}

Tableau 1 : Tableau des anniversaires à souhaiter

Cette connaissance est importante pour deux raisons.

- $\quad$ La première est la constitution des groupes de travail : suivant la matière et la taille des groupes, il est essentiel de penser la répartition des individus (connaissance de la matière, aisance dans la communication, prise de responsabilité, animosité...).

Comme plusieurs groupes peuvent travailler de front durant la même période, il est important de faire varier les membres des différentes équipes pour permettre :

- à chacun de travailler dans une équipe différente avec des personnalités diverses, c'est une expérience riche pour le travail en entreprise,

- de changer les chefs de groupe qui émergent automatiquement,

- d d'éviter la formation de sous-groupes à l'intérieur de la promotion.

- La seconde raison est le relais dans les cas d'urgence. En effet, quand un étudiant a un problème grave ou qu'il est démoralisé, le coordinateur peut faire appel à "son" réseau pour le seconder. Ce soutien, par les pairs, est rapide et efficace : mails, coups de téléphone plus fréquents, parfois visite ou organisation de soirées...

\subsection{Les outils avec plus de détails}

Pour les étudiants, la question de confiance est indispensable. Les étudiants doivent percevoir le coordinateur comme un appui autant sur le plan de la formation que privé. En effet, dans une formation à distance c'est la personne entière qui "entre" en formation, puisque son lieu de travail est son lieu privé (sa maison - son travail). Les problèmes domestiques influencent ainsi tous les aspects de la formation. 


\begin{tabular}{|c|c|c|c|c|c|c|}
\hline Prénom & starbox & & paces web & & GED & \\
\hline Cédric & molet & $p$ & violet & $p$ & lilas & $p$ \\
\hline Sabrina & jaune & $p$ & jaune & 0 & jonquille & $p$ \\
\hline Laëtitia & rowge & $p$ & rouge & $p$ & coquelicat & $p$ \\
\hline Estelle & blew & $p$ & blen & $p$ & ble uet & $p$ \\
\hline Marie & wert & $p$ & vert & 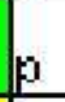 & menthe & $\mathrm{p}$ \\
\hline Danielle & wert & m & jaune & $m$ & caquelicat & m \\
\hline B rumo & jaune & $p$ & rouge & $p$ & menthe & $p$ \\
\hline Aude & wert & $\mathrm{g}$ & ذаแпе & p & blewet & 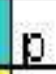 \\
\hline Lelia & rouge & m & vert & $\mathrm{m}$ & jonquille & m \\
\hline Patrice & rouge & $\mathrm{p}$ & bleu & $p$ & joncuille & $\mathrm{p}$ \\
\hline Pascal & jaune & $\mathrm{p}$ & vert & $p$ & ble uet & $\mathrm{p}$ \\
\hline David & molet & m & jaune & m & blewet & m \\
\hline Terry & molet & $\mathrm{p}$ & vialet & p & joncuille & $p$ \\
\hline Christine & blest & $\mathrm{p}$ & violet & $p$ & lilas & $p$ \\
\hline Patrick & molet & $p$ & rouge & $p$ & lilas & $p$ \\
\hline Charles & wert & $\mathrm{p}$ & violet & $\mathrm{p}$ & coquelicat & $\mathrm{D}$ \\
\hline Jear & blew & in & baleu & m & menthe & in \\
\hline Caroline & jaune & m & rouge & $m$ & lilas & m \\
\hline Claude & baleu & $\mathrm{p}$ & vert & $\mathrm{p}$ & coquelioat & $\mathrm{p}$ \\
\hline
\end{tabular}

Tableau 2 : Tableau des groupes de travail avec tri possible

On ne peut se voiler la face, la formation à distance multiplie les désorientations. En plus d'une remise cause, due à la reprise de toute formation, l'isolement est pesant, le face à face à la machine moins convivial que ne le pensent les informaticiens ${ }^{[5]}$, les ajustements de groupe manquent ${ }^{[6]}$, les repères temporels aussi. Pas de début ni de fin de cours qui en formation en face à face sont établis par l'institution. On oublie le temps devant son ordinateur, ou on se perd dans des détails. Certains jours, il faut avoir de la volonté et de la persévérance pour se mettre devant son PC, hors contexte de travail (salle de classe, bureau...), car les tentations sont grandes et à portée de main (télévision, frigo...). La perte de repères est réelle, même si elle varie selon les individus. C'est au coordinateur de remettre en place des repères, au fur à mesure que certains se désagrègent, par un suivi personnel constant, à la fois sur le plan psychologique et parfois social. Le téléphone reste à ce moment l'outil essentiel, celui qui transmet la voix humaine alors que le courriel est trop impersonnel. 


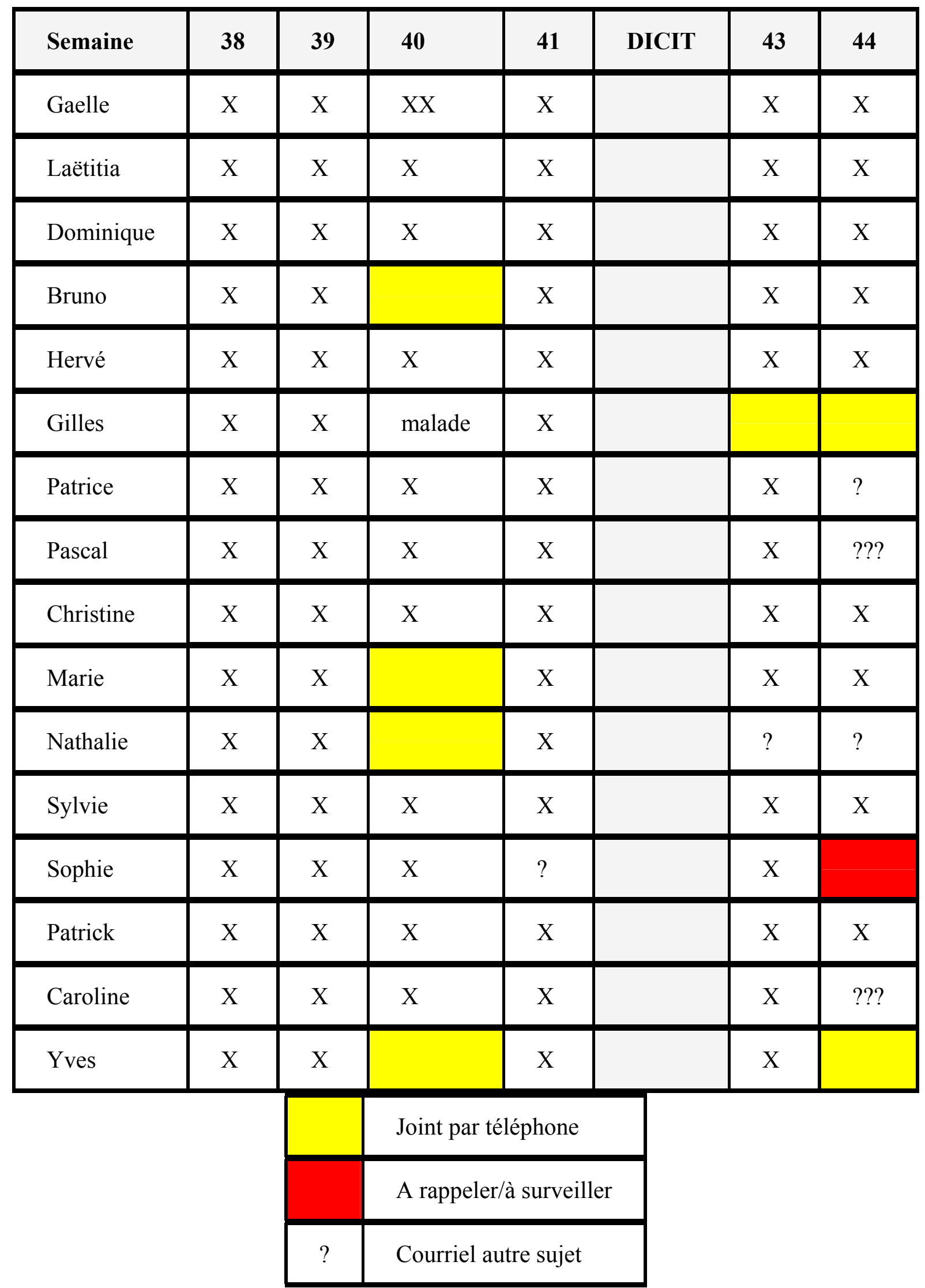

Tableau 3- Suivi des courriels du mardi (extrait) 
Un suivi régulier du travail s'établit avec des tableaux de suivi par matière, par session et par étudiant. La plupart du temps, ces tableaux sont à créer, puisque les outils de coordination ne sont pas prévus dans la conception même des plates-formes. Ces tableaux permettent de visualiser rapidement ceux qui "décrochent". Dans ce cas, le courriel est le premier media pour venir aux nouvelles. Si la réponse n'est pas assez rapide le téléphone prend le relais. Pour les exercices en groupe nécessitant des activités en forum, le coordinateur est parfois appelé pour régler les échanges et solliciter ceux qui restent en retrait.

Enfin, comme prévention à une démoralisation, un mail hebdomadaire est demandé à jour fixe sans sujet précis $^{[7]}$. Ce courriel défouloir permet de juger du moral des troupes : obligation est faite de répondre à tous personnellement. Ce mail sert donc à la fois à resserrer les liens (beaucoup d'étudiants se confient facilement par écrit) et à repérer les absents momentanés (les distraits : houps ! c'était jeudi ?, les occupés : désolé, nous sommes vendredi mais...) et ceux qui ont besoin de soutien. Mais même un courriel anodin peut être un appel au secours, il faut apprendre à déceler les indices entre les lignes, les fausses plaisanteries et les banalités. Faire le diagnostic des courriels est un travail de fond et les courriels du "SOS" au "RAS" obligent à bien connaître les interlocuteurs pour ne pas se tromper et anticiper la démotivation ou la désorientation.

Si le lendemain du jour fixé, un nom reste à l'index, il est nécessaire de téléphoner pour comprendre le problème et trouver rapidement une solution. Cette régulation à long terme permet d'assurer les meilleures conditions de travail, jusqu'à l'obtention du diplôme, car les étudiants se sentent soutenus.

\section{Conclusion}

La fonction de tuteur est essentielle à la formation à distance, si l'on prend comme postulat "la formation avec un accompagnement sur chaque matière, ses techniques et ses outils". Ce rôle de suiveur pédagogique permet alors à l'étudiant de progresser, de comprendre ses faiblesses et de les combler, d'avoir un interlocuteur qui connaît son sujet.

Dans la fiche d'ALGORA ${ }^{[8]}$ concernant les fonctions d'accompagnement, le tutorat est plus large. Certains points comme :

- $\quad$ Réaliser des opérations de bilan, validation des acquis professionnels, positionnement,

- Concevoir des parcours, des éléments de parcours ou des documents complémentaires

- Coordonner son travail avec celui d'autres tuteurs ou formateurs en fixant des règles d'organisation, en se faisant le relais des remarques et évaluations que les apprenants formulent ne rentrent pas dans les fonctions des tuteurs au DICIT.

Au DESS DICIT, le tutorat est plutôt rattaché à un contenu, à un domaine, pas à l'organisation. Ce rôle de tuteur est variable suivant les formations, il peut être plus ambitieux et couvrir une partie de la coordination. Nous avons préféré scinder les deux aspects du suivi, les tuteurs suivent les étudiants sur de petits parcours (deux à trois mois) et sur une matière, alors que le coordinateur les suit sur toute la formation et s'occupe de manager à la fois les tuteurs et les enseignants.

La coordination réalise un suivi plus étendu à la fois pédagogique, psychologique et social, mêlant un rôle de soutien à un rôle institutionnel. En effet, pour reprendre la fiche d'ALGORA, et faire un inventaire plus complet de la coordination. On peut dire que :

"La coordination recouvre un certain nombre de tâches qui incluent :

1. la communication institutionnelle,

2. la démarche qualité,

3. l'efficacité et l'homogénéisation de la formation,

4. l'évaluation du groupe et de son avancement,

5. la médiation et la régulation des conflits, 
6. le suivi des règles techniques et de civilité,

7. l'accroissement des compétences de l'équipe pédagogique,

8. l'organisation du travail des intervenants ponctuels,

9. la communication avec les entreprises,

10. le soutien de la synergie de groupe et le suivi du moral des troupes... "

L'accompagnement personnalisé a été, pour nous, un choix face à la volonté souvent affichée de parcours personnalisés. Le DESS DICIT en est à sa septième promotion, les rôles ainsi définis conviennent à notre projet et notre organisation. Certains les diront contraires à l'autonomie des étudiants, l'expérience nous montre pourtant que cette prise en charge, choisie et affichée, respecte leur personnalité et les aide à se structurer à distance.

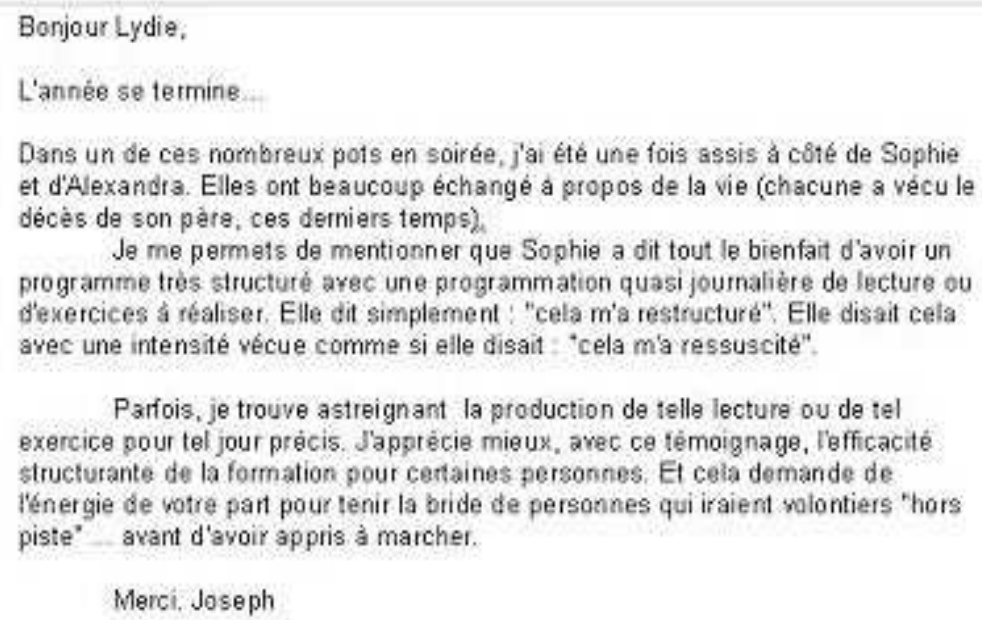

Figure 4 : Courriel d'un ancien étudiant à la fin de sa formation

\section{Références}

\section{Bibliographie}

\section{[Blandin02]}

Blandin. B. (2002). Les mondes sociaux de la formation. Éducation permanente. Les TIC au service des nouveaux dispositifs de formation. $\mathrm{N}^{\circ} 152$. pp 199-211.

\section{[Helson03]}

Helson. C. (2003). Être accompagné dans ses états d'âge : l'effet d'accentuation des dates anniversaires. Éducation permanente. L'accompagnement dans tous ses états. $\mathrm{N}^{\circ} 153$. pp 69-77.

\section{[LeBouëdec03]}

Le Bouëdec.G. (2003). La démarche d'accompagnement, un signe des temps. Éducation permanente. L'accompagnement dans tous ses états. $\mathrm{N}^{\circ} 153$. pp 13-18.

\section{Bibliographie Web complémentaire}

\section{[Algora03a]}

Algora (anciennement Oravep). (2003). Étude comparative technique et pédagogique des plates-formes pour la formation ouverte et à distance. http://www.educnet.education.fr/superieur/plateforme.htm 


\section{[Algora03b]}

Algora. (2003). Mise en auvre des plates-formes pour la formation ouverte et à distance : les fonctions d'accompagnement. http://ressources.algora.org/ressources/environnements/lms/pfm.asp

\section{[Chasseneuil03]}

Collectif de Chasseneuil. (2003). Formations ouvertes et à distance : l'accompagnement pédagogique et organisationnel. http://ressources.algora.org/reperes/comprendre/chasseneuil/tel.asp

\section{[Jaudeau03]}

Jaudeau, M. (2003). Comment fidéliser les cyberétudiants? Quelques points essentiels. http://thot.cursus.edu/rubrique.asp?no=16764.

\section{[Paquette03]}

Paquette D. (2003). Le rôle des tuteurs et des tutrices : une diversité à appréhender. Revue du Conseil Québécois de la Formation à http://cqfd.teluq.uquebec.ca/Activites/DistanceS/dist_ant/Vol5N1/vol5n1.html

\section{[Pettingrew03]}

Pettingrew F. (2003). L'encadrement des cours à distance : profils étudiants. Revue du Conseil Québécois de la Formation à $\quad$ Distance. http://cqfd.teluq.uquebec.ca/Activites/DistanceS/dist ant_Vol5N1/vol5n1.html

\section{[Bernatchez03]}

Bernatchez, P.A. (2003). L'encadrement des apprenants par la télématique. DistanceS. 2,2, pp 65-79. http://cqfd.teluq.uquebec.ca/IN_Auteurs/in_auteurs.html

\section{[BéginGagné03]}

Bégin J et Gagné P. J. (2003). L'encadrement des études à distance par des personnes tutrices : qu'en pensent les étudiants ?. $\quad$ DistanceS. 5, http://cqfd.teluq.uquebec.ca/IN_Auteurs/in_auteurs.html

\section{[DionneMercier03]}

Dionne, M., and Mercier, J. (2003). Profil des activités d'encadrement comme soutien à l'apprentissage en formation à distance. Revue du Conseil Québécois de la Formation à Distance 3, 2, pp 69-99. http://cqfd.teluq.uquebec.ca/IN_Auteurs/in_auteurs.html

\section{[Hill03]}

Hill, P. (2003). Comment étendre la portée de la formation à distance sans sacrifier le sens des relations

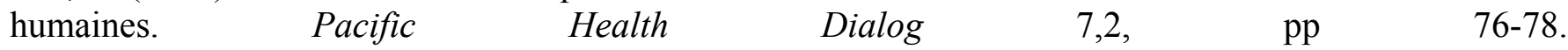
http://www.spc.int/phs/Francais/FR/publications/PHD/PHD-FR-pages76-78.pdf

\section{[Lameule00]}

Lameule, G. 2000. Former et échanger par les réseaux. http://www.edusud.org/ressources/ingenierie/foad.doc

\section{[Lebel03]}

Lebel, C. (2003). Plaisir des mots: L'intervention auprès de l'étudiant à distance peut-elle être douloureuse et dangereuse ? Revue du Conseil Québécois de la Formation à Distance 4, 2, pp 75-78. http://cqfd.teluq.uquebec.ca/IN_Auteurs/in_auteurs.html 


\section{[MinistèreDGEFP03]}

Ministère de l'emploi et de la solidarité, Délégation Générale à l'Emploi et à la Formation Professionnelle. (2003). Circulaire DGEFP $\mathrm{N}^{\circ} 2001 / 22 \mathrm{du} 20$ juillet 2001 relative aux formations ouvertes et/ou à distance "FOAD". http://ressources.algora.org/reperes/reglementation/textes/dgefp0122.asp

\section{[Morrison03]}

Morrison, D. (2003).. Project GOLD : Supporting Distance Learning Students. Ariadne, http://www.ariadne.ac.uk/issue28/gold/

\section{A propos des auteurs}

Lydie Réné-Boullier est formatrice en mathématiques, elle a repris des études en Sciences de l'éducation à Amiens, puis en Communication à Paris XIII. Ingénieur d'études à l'Université de Technologie de Compiègne, elle a été chef de projet organisationnel du Campus Numérique Insecte. Elle assure la coordination pédagogique du DESS DICIT depuis 7 ans. Elle s'intéresse à l'accompagnement global des personnes apprenantes et aux raisons de l'abandon.

Adresse : Laboratoire

Université

BP 60319-60203 Compiègne,

d'Ingénierie

Technologie
Pédagogique

de

Courriel : 1ydie.rene-boullier@utc.fr

[1] "L'accompagnement se fait toujours à l'occasion d'une fonction d'autorité, donc au sein d'une institution, et en référence aux finalités, aux valeurs et aux critères de celle-ci.... Ne plus s'en remettre à d'autres qui pensent pour lui. Cette situation, sans doute exaltante pour l'autonomie, est en réalité angoissante sinon désespérante, tant l'incertitude est grande... voilà le contexte dans lequel surgissent assez naturellement le besoin et l'offre d'accompagnement..." [LeBouëdec02].

[2] Recherche en cours dans le cadre d'un DEA : "La désorientation dans l'apprentissage à distance, comme moment de recréation de compromis entre principes industriel et domestique" (nom provisoire), Laboratoire Costech à l'Université de Technologie de Compiègne.

[3] Aujourd'hui le cursus est de 16 unités de valeurs et 19 enseignants/tuteurs et il accueille aussi des apprentis.

[4] Dans les captures d'écran, les noms ont été cachés ou seuls les prénoms sont utilisés. Pour les tableaux, nous avons transformé les prénoms rares pour en mettre de plus usités.

[5] L'informatique est un pré-requis de base pour les formations à distance et il reste souvent un obstacle. Les personnes débutantes hésitent quand elles sont seules -elles ont peur de faire une bêtise et cela les paralysent.

[6] Lors d'un cours en face à face, la réponse à une question est utile à toute la promotion. De plus, le fait de voir ses collègues poser des questions permet à l'étudiant reprendre confiance en ses capacités (je ne suis pas le seul à ne pas avoir tout compris...).

[7] Il s'agit là d'un équivalent de la fonction phatique du langage analysée par Jakobson dans son modèle. (Essais de linguistique, éditions de minuit). 
[8] Algora - Formations Ouvertes et Réseaux. Association soutenue par le Ministère des Affaires Sociales, du Travail et de la Solidarité (par le biais de la Direction Générale de l'Emploi et de la Formation Professionnelle), qui a pour objet d'accompagner et de promouvoir le développement de la FOAD et l'usages des TIC dans les systèmes de Formation professionnelle. Les fiches fournissent, aux concepteurs de dispositif de formation à Distance, des éléments permettant de dimensionner et d'organiser un projet.

\section{Référence de l'article :}

Lydie Réné-Boullier, Coordination pédagogique : une vision globale de l'accompagnement personnalisé au DESS DICIT, Revue STICEF, Volume 10, 2003, ISSN : 1764-7223, mis en ligne le 5/02/2004, http://sticef.org

(C) Revue Sciences et Technologies de 1'Information et de laCommunication pour l'Éducation et la Formation, 2003 